\title{
Nanotecnologia farmacêutica aplicada ao tratamento da malária
}

\author{
Lúcio Figueira Pimentel ${ }^{1}$, Agenor Tavares Jácome Júnior ${ }^{1}$, Vanessa Carla Furtado Mosqueira ${ }^{3}$, \\ Nereide Stela Santos-Magalhães ${ }^{1,2^{*}}$
}
'Laboratório de Imunopatologia Keizo Asami, Universidade Federal de Pernambuco, ${ }^{2}$ Departamento de Bioquímica, Universidade Federal de Pernambuco, ${ }^{3}$ Departamento de Farmácia, Universidade de Ouro Preto.

\footnotetext{
* Correspondência:

N. S. Santos Magalhães

Universidade Federal de Pernambuco -UFPE

Grupo de Sistemas de Liberação

Controlada de Medicamentos

Laboratório de Imunopatologia Keizo Asami - LIKA

Av. Prof. Moraes Rego, 1235, Cidade Universitária.

50670-901 - Recife-PE - Brasil

E-mail: nssm@ufpe.br
}

Apesar do desenvolvimento tecnológico e científico, a malária permanece como um dos maiores problemas de saúde a serem combatidos. As estratégias modernas para o controle da doença prevêem ações conjuntas, como o combate do inseto vetor, diagnóstico rápido e preciso, garantia de terapêtica adequada, redução dos casos de resistência, além do desenvolvimento de novos agentes terapêticos e vacina e através da otimização da ação de fármacos utilizados na atualidade. Os sistemas de liberação controlada de fármacos vêm recebendo atenção especial nesta área de pesquisa, com o desenvolvimento de estratégias para a veiculação de agentes bioativos e vacinas na forma de nanodispositivos tais como lipossomas, nanopartículas e micropartículas. Diversos nanossistemas já demonstraram eficácia na otimização de vacinas e quimioterápicos destinados ao controle da malária. Este artigo de revisão tem por objetivo avaliar o estado da arte na terapêutica da malária e demonstrar o potencial da nanotecnologia farmacêutica como ferramenta destinada ao combate da doença.
Unitermos

- Malária

- Nanotecnolgia

- Lipossomas

- Nanopartículas

- Micropartículas

- Vacinas

\section{INTRODUÇÃO}

A malária humana, uma doença parasitária que tem como agentes etiológicos quatro espécies de protozoários do gênero Plasmodium (P. vivax, P. ovale, P. malariae e P. falciparum), é transmitida ao homem através da picada de fêmeas do inseto do gênero Anopheles (Krettli et al., 2001), sendo a infestação humana mais devastadora no mundo inteiro, com 300 a 500 milhões de casos clínicos e quase 3 milhões de óbitos a cada ano (Gomes et al., 2001; Tracy, Webster Júnior, 2003).
Segundo Winstanley (2001), as falhas no tratamento da malária são devidas a múltiplos fatores. A complexidade dos esquemas implementados, que consistem geralmente em dois ou mais medicamentos, dificulta a adesão do paciente. $\mathrm{O}$ baixo índice terapêutico dos antimaláricos e algumas interações medicamentosas restringem a aplicação em alguns casos, comprometendo a eficácia do tratamento. Adicionalmente, falhas técnicas e operacionais na execução de campanhas de combate à doença, aliadas à redução de recursos financeiros governamentais a elas destinados, à resistência do vetor aos inseticidas utilizados, à re- 
sistência do Plasmodium aos antimaláricos e às condições sócio-econômicas das populações atingidas, agravam as dificuldades para a erradicação da malária no mundo (Dias, 2002).

O uso racional de fármacos no combate à malária tornou-se crucial, pois a seleção adequada dos antimaláricos está diretamente relacionada ao futuro das políticas de combate, à prevenção de epidemias e ao controle da morbidade e mortalidade da doença (Wongsrichanalai et al., 2002).

Pesquisas de novos compostos antimaláricos, desenvolvimento de uma vacina eficaz, além da nanotecnologia farmacêutica aplicada ao desenvolvimento de vacinas e medicamentos antimaláricos em nanossistemas, constituem-se em estratégias de valia na erradicação da malária.

Diversas pesquisas vêm demonstrando o grande potencial da nanotecnologia farmacêutica no tratamento, prevenção e diagnóstico de inúmeras patologias, dentre elas as parasitárias. Nesse contexto, técnicas inovadoras estão sendo aplicadas na obtenção de novas formas farmacêuticas de liberação controlada de fármacos capazes de manter ou ampliar a ação de agentes promissores utilizados no combate e controle da malária (Chedgzoy et al., 2002; Chimanuka et al., 2002). Nanodispositivos permitem o uso de antimaláricos potencialmente tóxicos e pouco utilizados devido a estas características (Mosqueira et al., 2004), bem como o aumento na duração e eficácia da resposta imune a vacinas (Alving, 2002; Carcaboso et al., 2003).

Esta revisão tem como objetivo avaliar o estado da arte na terapêutica antimalárica e demonstrar o potencial da nanotecnologia farmacêutica como ferramenta destinada ao combate e controle da malária. Para tanto, abordamse questões relevantes sobre a doença, tais como, medidas de prevenção, diagnóstico, terapêutica convencional e as alternativas nanotecnológicas para novas formas farmacêuticas contendo antimaláricos e vacinas.

\section{Malária}

A malaria é uma doença infecciosa causada por um protozoário unicelular do gênero Plasmodium, podendo ser transmitida para o ser humano pela picada do mosquito do gênero Anopheles, por transfusão de sangue ou compartilhamento de agulhas e seringas infectadas com o parasita (Krettli et al., 2001).

A malária ocorre com maior freqüência nos países tropicais, onde quatro espécies infectam o homem: $P$. falciparum, $P$. vivax, P. malariae e $P$. ovale (Foley, Tilley, 1998; Krettli et al., 2001). Cada espécie de Plasmodium produz um padrão de doença bastante característico, relacionado, em parte, com o momento de seu ciclo intra- eritrocítico assexuado. As infecções por Plasmodium vivax e $P$. ovale raramente são fatais e caracterizam-se por picos febris com intervalos de cerca de 48 horas (malária terçã benigna). Com o P. malariae, os picos febris ocorrem em intervalos de 72 horas (malária quartã benigna) (Kumar et al., 2005; FUNASA, 2001). Por outro lado, a infecção causada pelo $P$. falciparum é responsável pelas mais altas parasitemias e pela maior parte da mortalidade. $\mathrm{O}$ ataque agudo de malária caracteriza-se por um conjunto de paroxismos febris, que apresentam quatro períodos sucessivos: o de frio, calor, suor e apirexia (Tracy, Webster Júnior, 1996).

No Brasil, prevalecem as infecções causadas pelo $P$. vivax e $P$. falciparum (Kumar et al., 2005; FUNASA, 2002a,b). A natureza malígna do P. falciparum pode estar relacionada com o fato de que a parasitemia ocorre indiscrimi-nadamente em todas as hemácias com receptores para glicoforina, resultando em parasitemias elevadas, que se instalam bruscamente. Adicionalmente, o $P$. falciparum induz a formação de proeminências nas membranas dos eritrócitos, responsáveis pela adesão ao endotélio dos microvasos. Esse processo causa obstrução vascular, hipoxia tecidual e/ou necrose isquêmica, especialmente em crianças de até 5 anos, produzindo a sintomatologia conhecida como malária cerebral (Coltel et al., 2004). Essa forma freqüentemente letal da doença caracteriza-se pelos seguintes elementos: acometimento cerebral com delírio evoluindo para o coma, choque hipotérmico (malária álgida), edema pulmonar agudo, coagulação intravascular disseminada, insuficiência renal com hemoglobinúria e insuficiência hepática (Kumar et al., 2005). Em todas as manifestações, a ruptura das hemácias resulta em anemia e, ao mesmo tempo, é liberado o pigmento malárico (produto da digestão do grupo heme por parte do parasita). As hemácias lesadas e o pigmento malárico são removidos do sangue pelo sistema fagocitário mononuclear, induzindo, assim, esplenomegalia e hepatomegalia.

\section{Terapêutica}

A quimioterapia é o principal fator para a redução da morbidade e mortalidade relacionada à malária, segundo Kremsner e Krishna (2004). A tomada de decisão para o tratamento adequado de um paciente com malária deve ser precedida de informações sobre a gravidade da doença, espécie de plasmódio, idade do paciente, histórico de exposição anterior à infecção e a suscetibilidade dos parasitas aos antimaláricos convencionais (Tabela I e II). Atualmente, tratamento único igualmente efetivo contra ambas as espécies de plasmódios prevalentes em nosso país ( $P$. vivax 
TABELA I - Classificação de antimaláricos de acordo com sua categoria química e mecanismo de ação (adaptado de FUNASA, 2001)

\begin{tabular}{lcc}
\hline Composto & Categoria Química & Mecanismo de ação \\
\hline Cloroquina e amodiaquina & 4 -aminoquinolina & Digestão de produtos da hemoglobina \\
Primaquina & 8 -aminoquinolina & Inibe a respiração mitocondrial do parasita \\
Quinina, mefloquina e halofantrina & quinolinametanóis e & Digestão de produtos da hemoglobina \\
& fenantrenometanol & \\
Artemisinina e seus derivados & Éter de lactona sesquiterpênica & Metabolismo das proteínas do parasito \\
Tetraciclina e doxiciclina & Derivados de naftaceno & Síntese das proteínas do parasito \\
Clindamicina & Lincosaminas & Síntese das proteínas do parasito \\
\hline
\end{tabular}

e P. falciparum) ainda não foi estabelecido. Para cada espécie de plasmódio, vem sendo utilizado um medicamento ou associações de medicamentos específicos, em dosagens adequadas à situação particular de cada doente.

Entre os agentes terapêuticos eficazes com ação contra os parasitas da malária estão as famílias das quinolinas (quinina, cloroquina, primaquina, mefloquina, amodiaquina, halofantrina), diclorobenzilidina (lumefantrina), biguanidas (proguanil, clorproguanil), diaminopiridinas (pirimetamina), sulfonas (dapsona), hidroxinaftoquinonas (atovaquona) e lactonas sesquiterpênicas (derivados da artemisinina, artesunato e artemeter) segundo Loiseau e Le Bras (2007). Os antimaláricos são rotineiramente utilizados em associação com outros quimioterápicos tais como sulfonamidas (sulfadoxina), tetraciclinas (tetraciclina, doxicilina) e lincosaminas (clindamicina e lincomicina). Estes agentes terapêuticos apresentam muitos inconvenientes relacionados ao seu uso, pois eles compreendem complexos regimes de administração e muitos efeitos colaterais, o que contribui para interrupção do tratamento e o possível desenvolvimento de resistência pelo parasita (Crawley, 1999; Winstanley, 2001). O tratamento adequado e oportuno da malária constitui hoje o principal alicerce para o controle da doença.

A resistência do Plasmodium aos antimaláricos é considerada o maior problema no controle da malária (Crawley, 1999). O P. falciparum desenvolveu resistência à cloroquina e à 4-aminoquinolina há mais de 50 anos. Atualmente, a resistência do $P$. falciparum se estende a muitos antimaláricos, incluindo a sulfadoxina associada à pirimetamina e à mefloquina, determinando o aparecimento de áreas em que a multi-resistência a fármacos é predominante (Wongsrichanalai et al., 2002). O desenvolvimento de multi-resistência aos antimaláricos determina a urgência de novos regimes de tratamento visando ao controle da propagação da doença. Para estes autores, a combinação de antimaláricos pode ser utilizada para o tratamento de infecções de $P$. falciparum multi-resistentes. Com o surgimento da resistência múltipla a fármacos pelo $P$. falciparum, no- vos fármacos e combinações de fármacos são urgentemente necessários para o controle da malária. Pesquisas com inibidores da protease MSP-1, combinação de antifolatos de terceira geração, inibidores da biossíntese de ácidos graxos, inibidores da lactato desidrogenase, além de inibidores do metabolismo de fosfolipídios e inibidores de transferase, como a proteína farnesil de $P$. falciparum, estão sendo realizadas para o desenvolvimento de novos antimaláricos (Wilairatana et al., 2002). O seqüenciamento do genoma do Plasmodium permite a identificação de alvos potenciais para o desenvolvimento rápido de novos fármacos de baixo custo para utilização no controle da malária (Florens et al., 2002).

A toxicidade induzida pelo uso da maioria dos antimaláricos utilizados no tratamento e na profilaxia da malária é outro problema a ser minimizado para garantir a observância do paciente e assegurar o controle adequado da doença. Os antimaláricos freqüentemente causam efeitos gastrintestinais adversos sem gravidade clínica, mas que irritam o paciente, tais como náuseas e vômitos causados pelo uso de mefloquina ou o prurido causado pelo uso de cloroquina, comprometendo a adesão do paciente e a eficácia do regime adotado. Efeitos mais graves podem ser observados quando o tratamento ocorre em dose única (Winstanley, 2001).

O desenvolvimento de novos regimes de utilização de antimaláricos em combinação e o uso de informações moleculares, obtidas dos estudos de seqüenciamento do genoma de plasmódios, serão de vital importância para a identificação de alvos para o desenvolvimento de novas formas farmacêuticas sítio-específico contendo antimaláricos.

\section{NANOTECNOLOGIA FARMACÊUTICA APLICADA AO TRATAMENTO DA MALÁRIA}

A nanotecnologia farmacêutica é a área das ciências farmacêuticas envolvida no desenvolvimento, caracterização e aplicação de sistemas terapêuticos em escala nanométrica ou 
TABELA II - Fármacos antimaláricos: vantagens e desvantagens de seu uso clínico

\begin{tabular}{lll}
\hline Composto & Vantagens & Desvantagens \\
\hline Cloroquina & $\begin{array}{l}\text { Rápida atividade esquizonticida; atividade } \\
\text { gametocitocida para } P \text {. vivax e } P \text {. malariae; ação } \\
\text { antipirética e antiinflamatória }\end{array}$ & $\begin{array}{l}\text { Poucas cepas de P. falciparum são, ainda, sensíveis à } \\
\text { cloroquina }\end{array}$
\end{tabular}

Amodiaquina

Primaquina

Quinina

Mefloquina

Halofantrina

Artemisinina e derivados

Tetraciclina

Doxiciclina

Clindamicina
Rápida atividade esquizonticida; atividade gametocitocida para $P$. vivax e P. malariae; ação antipirética e antiinflamatória

Ação profilática causal, sendo altamente ativa contra gametócitos de todas as espécies de plasmódios e contra hipnozoítos do $P$. vivax

Eficaz contra $P$. falciparum (fármaco de escolha para a malária falciparum em associação com doxiciclina ou tetraciclina)

Potente esquizonticida sanguíneo, altamente ativo contra P.vivax e P. malariae; eficaz contra os gametócitos de $P$. vivax

Ação esquizonticida sanguínea sobre todas as espécies de plasmódio

Esquizonticidas sanguíneos potentes de ação rápida

Antimicrobiano de amplo espectro com ação potente contra as fases sanguíneas e intrahepáticas do P. falciparum (em combinação com a quinina)

Difere das tetraciclinas pelo fato de ser mais completamente absorvida, mais lipossolúvel e meia vida mais longa; usada no tratamento da malária por $P$. falciparum

Antimicrobiano de amplo espectro esquizonticida sanguíneo eficiente; tratamento da malária por $P$. falciparum
P. falciparum já é resistente em todas as áreas endêmicas do mundo

Alta toxidade em uso prolongado necessário para atuar contra as fases assexuadas sanguíneas; contraindicada na gravidez (hemólise em fetos) e para crianças menores de 6 anos (hipoplasia e aplasia medular)

Alta capacidade de se ligar a proteínas; distribui-se por todos os fluidos do corpo; atravessa a barreira placentária e a hematoencefálica; necessidade de uso associado a outros fármacos, devido à baixa adesão quando em uso isolado; muitos efeitos colaterais (chinchonismo)

Alta capacidade de ligação à proteínas; meia vida muito longa (10-40 dias); esofagite após ingestão; potencial para induzir manifestações neuropsiquiátricas graves

Não atua sobre gametócitos e hipnozoítos; custo elevado; variabilidade de sua biodisponibilidade; resistência cruzada com a mefloquina; cardiotoxidade em certos grupos de risco após dosagem padrão; não deve ser usada por gestantes ou lactentes

Neurotoxidade dose/dependente

Contra-indicado na gravidez e em crianças menores de 8 anos, pois prejudica a calcificação óssea no feto, pode resultar em osteogênese anormal e hipoplasia do esmalte dentário, atravessam a barreira placentária e são encontradas no leite materno; deve ser sempre empregada em combinação com outro antimalárico

Mesmas contra-indicações da tetraciclina; deve ser sempre empregada em combinação com outro agente antimalárico

Mais cara e mais tóxica que doxiciclina e tetraciclina; deve ser sempre empregada em combinação com outro agente antimalárico micrométrica. Estudos de tais sistemas têm sido realizados ativamente no mundo com o propósito de direcionar e controlar a liberação de fármacos (Sakata et al., 2007). A aplicação da nanotecnologia para o tratamento, diagnóstico, monitoramento e controle de sistemas biológicos foi recentemente denominada "Nanomedicina" pelo National Institute of 
Health nos Estados Unidos (Moghimi et al., 2005). Esta tecnologia surgiu nos anos 1960 com o desenvolvimento inicialmente da microencapsulação, técnica de transformação de líquidos (polímeros e outras substâncias) em pós com tamanho de partículas micrométricas. A microencapsulação é bastante utilizada nas indústrias alimentícia, têxtil, farmacêutica e cosmética por permitir a proteção de substâncias lábeis e voláteis, o controle da liberação do fármaco, contribuindo para a melhoraria na biodisponibilidade e redução da dose terapêutica e toxicidade. Amicroencapsulação serviu de modelo para técnicas mais sofisticadas, agora em escala nanométrica, permitindo o desenvolvimento de nanopartículas. A descoberta dos lipossomas nos anos 1960 veio aumentar a variedade de ferramentas para o desenvolvimento da nanotecnologia farmacêutica com sistemas lipídicos para vetorização de fármacos (Lasic, 1998). Atualmente são desenvolvidos nanossistemas, tais como lipossomas e nanopartículas, e microssitemas, como micropartículas, emulsões multiplas e microemulsões (Silva, 2004). Lipossomas são vesículas aquosas circundadas por bicamada lipídica podendo servir como veículo de fármacos a serem encapsulados na cavidade aquosa da vesícula ou na bicamada lipídica (Lasic, 1998). Nanopartículas são partículas poliméricas na forma de reservatório (cápsulas) ou matricial (matriz polímerica) nas quais o fármaco está encapsulado ou adsorvido na malha polimérica (Brigger et al., 2002). Nos anos 1990 surgiram nanossistemas mais sofisticados revestidos por polímeros hidrofílicos, denominados sistemas furtivos, que permitem um tempo de circulação maior no organismo (Gref et al., 1994). Além dos sistemas furtivos, sistemas contendo moléculas sinalizadoras na superficie denominados sítio-específicos foram desenvolvidos com a finalidade do direcionamento específico de fármacos para células alvo (Barrat, 2000).

Entre as vantagens que os nanossistemas podem oferecer destacam-se: a proteção do fármaco no sistema terapêutico contra possíveis instabilidades no organismo, promovendo manutenção de níveis plasmáticos em concentração constante; o aumento da eficácia terapêutica; a liberação progressiva e controlada do fármaco pelo condicionamento a estímulos do meio em que se encontram (sensíveis a variação de $\mathrm{pH}$ ou de temperatura); a diminuição expressiva da toxicidade pela redução de picos plasmáticos de concentração máxima; a diminuição da instabilidade e decomposição de fármacos sensíveis; a possibilidade de direcionamento a alvos específicos (sítioespecificidade); a possibilidade de incorporação tanto de substâncias hidrofílicas quanto lipofílicas nos dispositivos; a diminuição da dose terapêutica e do número de administrações e aumento da aceitação da terapia pelo paciente. Embora estas vantagens sejam significativas, alguns inconvenientes plausíveis não podem ser ignorados, como por exemplo, uma possível toxicidade, ausência de biocompatibilidade dos materi- ais utilizados e o elevado custo de obtenção dos nanossistemas comparados com as formulações farmacêuticas convencionais (Verma, Garg, 2001; Dunne et al., 2003; Tao, Desai, 2003).

\section{Lipossomas}

Lipossomas, vesículas aquosas formadas por bicamadas concêntricas de fosfolipídios (Figura 1), são uma excelente forma de sistema de liberação controlada de fármacos devido à sua flexibilidade estrutural (tamanho, composição e fluidez da bicamada lipídica), como à sua capacidade de incorporar variedade de compostos hidrofílicos (Andrade et al., 2004) e hidrofóbicos (Pontes et al., 1999), sendo os sistemas nanométricos mais estabelecidos clinicamente para a entrega de farmacos citotóxicos, genes e vacinas (Diebold et al., 2006). Os lipossomas podem ter tamanhos variados ( $20 \mathrm{~nm}$ até alguns micrometros) com espessura de bicamada lipidica em torno de $6 \mathrm{~nm}$. Os lipossomas como sistemas de liberação controlada de fármacos não apenas possibilitam a vetorização e a proteção do fármaco, como também permitem o possível direcionamento para sítios específicos de células ou órgãos (sítio-específicos) (Bergstrand et al., 2003; Sapra, Allen, 2003).

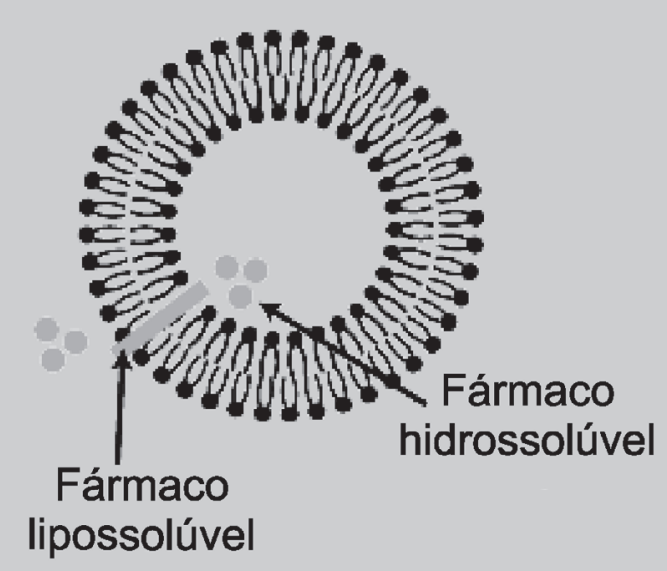

FIGURA 1 - Esquema ilustrativo do corte transversal de um lipossoma, que pode conter fármacos hidrofílicos na cavidade aquosa interna, enquanto fármacos hidrofóbicos ficam retidos na bicamada lipídica (modificado de http:// www.endovasc.com/imagens/graphics/liposomel.gif).

Bayomi e colaboradores (1998) desenvolveram formulações lipossômicas contendo artemeter, uma substância semisintética, derivada de artemisinina, efetivo contra os estágios eritrocíticos de $P$. falciparum resistente à cloroquina e à mefloquina (Brossi et al., 1988). Dentre as formulações desen- 
volvidas, aquela composta por dipalmitoilfosfatidilcolina (DPPC), diberenoilfosfatidilcolina (DBPC), colesterol e artemeter na proporção de 1:1:2:1, foi a selecionada para ensaios in vivo, utilizando coelhos machos "Nova Zelândia", que receberam os lipossomas por via oral e intravenosa. Os resultados mostraram aumento significativo da biodisponibilidade do fármaco, quando administrado em lipossomas por via oral (97,91\%), comparado com os $31,83 \%$ quando administrado sob a forma de suspensão oral. O artemeter em lipossomas administrados por via intravenosa resultou em aumento no tempo de meia-vida, quando comparado com aqueles de outros derivados da artemisinina, como a própria artemisinina e o artemeter.

$\mathrm{O}$ antimalárico artemeter foi encapsulado em lipossomas compostos de fosfatidilcolina de ovo e colesterol, sendo caracterizados quanto à sua capacidade de encapsulamento, estabilidade química e sua eficácia terapêutica em camundongos infectados pelo $P$. chabaudi. Uma eficiência de encapsulamento próxima a $100 \%$ foi observada com o fármaco localizado na bicamada lipídica e estabilidade de três meses, quando estocados a $4{ }^{\circ} \mathrm{C}$. A formulação demonstrou ainda eficácia terapêutica de $100 \%$ de cura após 22 dias de infecção (Chimanuka et al., 2002).

Lipossomas contendo artesunato, outro derivado da artemisinina, foram desenvolvidos à base de fosfatidilcolina de ovo e colesterol. Na caracterização da nova forma farmacêutica, foram realizados ensaios de microscopia, capacidade de incorporação e estabilidade química, além do perfil de liberação do fármaco a partir dos lipossomas. Os resultados evidenciaram a obtenção de um sistema estável por 10 dias a $25^{\circ} \mathrm{C}$, contendo $1 \mathrm{mg}$ de artesunato e $300 \mathrm{mg}$ de lipídios por $\mathrm{mL}$ de tampão com $\mathrm{pH} 5,0$ e taxa de encapsulamento de 100\% (Gabriels, Plaizier-Vercanmen, 2003).

Um 19-amino peptídeo extraído de uma proteína do esporozoíto de $P$. berghei foi preparado e ligado quimicamente à superfície de lipossomas furtivos de fosfatidilcolina marcados com lipídeos fluorescentes (Longmuir et al., 2006). Os resultados de biodistribuição indicaram a acumulação desses lipossomas pilotados nos hepatócitos e nas células não-parenquimais do fígado, centenas de vezes maior do que no coração, pulmão, rins e dez vezes maior que no baço. Esses resultados indicam que é possivel direcionar fármacos incorporados nesse tipo de lipossomas para o combate as formas do parasita no interior dos hepatócitos.

\section{Nanocápsulas}

As nanocápsulas são sistemas coloidais vesiculares de tamanho nanométrico, em que o fármaco está confinado em uma cavidade oca ou oleosa, estabilizada por mem- brana polimérica (Legrand et al., 1999; Brigger et al., 2002). As nanocápsulas são utilizadas para vetorização de fármacos hidrofóbicos, que são incorporados na cavidade interna oleosa (Santos et al., 2005).

Mosqueira e colaboradores $(2004,2006)$ desenvolveram formulações de nanocápsulas contendo halofantrina para a avaliação da eficiência e farmacocinética em estudos com camundongos infectados pelo $P$. berghei. Neste trabalho foram utilizadas nanocápsulas furtivas de polímero de ácido lático, com superfície modificada por polietilenoglicol, com a finalidade de reduzir a captura pelo sistema fagocitário mononuclear e proporcionar o aumento do tempo na circulação sanguínea. As nanocápsulas demonstraram a capacidade de modificar o perfil farmacocinético da halofantrina no plasma, mantendo as concentrações plasmáticas do fármaco por mais de $70 \mathrm{~h}$, indicando aumento no tempo de circulação das nanocápsulas furtivas. As nanocápsulas induziram rápido controle do desenvolvimento dos parasitas nas primeiras 48 horas pós-tratamento, com resultados estatisticamente significativos comparados ao controle. Os resultados mostraram que a nanoencapsulação da halofantrina promoveu perfil farmacocinético mais favorável e reduziu a cardiotoxicidade (Leite et al., 2007), sugerindo o uso de halofantrina nanoencapsulada, por via parenteral, para o tratamento da malária grave com menores efeitos adversos.

Bhadra e colaboradores (2006) desenvolveram nanopartículas baseadas em dendrímeros de PEG-poli-Llisina recobertas por sulfato de condroitina. $\mathrm{O}$ fosfato de cloroquina foi encapsulado e as partículas apresentaram reduzida capacidade hemolítica, reduziram o nível de trofozoítos jovens em cultura de $P$. falciparum e prolongaram a permanência da cloroquina no sangue de ratos em estudos de biodistribuição, após administração intravenosa, sem que efeitos adversos tenham sido observados.

\section{Micropartículas}

Micropartículas são partículas poliméricas esféricas matriciais (microesferas) ou reservatórios (microcápsulas) (Figura 2), que promovem a liberação controlada de fármacos ou substâncias biologicamente ativas hidrofílicas (Ahsan et al., 2002) ou hidrofóbicas (Ribeiro-Costa et al., 2004). O processo de microencapsulação possui larga aplicação industrial e para desenvolvimento de novas formas farmacêuticas devido às suas características de biocompatibilidade e biodegradabilidade (Mandal et al., 2001; Vandenberg et al., 2001; Youan et al., 2001).

Schlicher e colaboradores (1997) desenvolveram microesferas de co-polímero de ácido lático e glicólico (PLGA) contendo desferrioxamina (DFO), um agente quelante de ferro com atividade antimalárica in vitro e in 


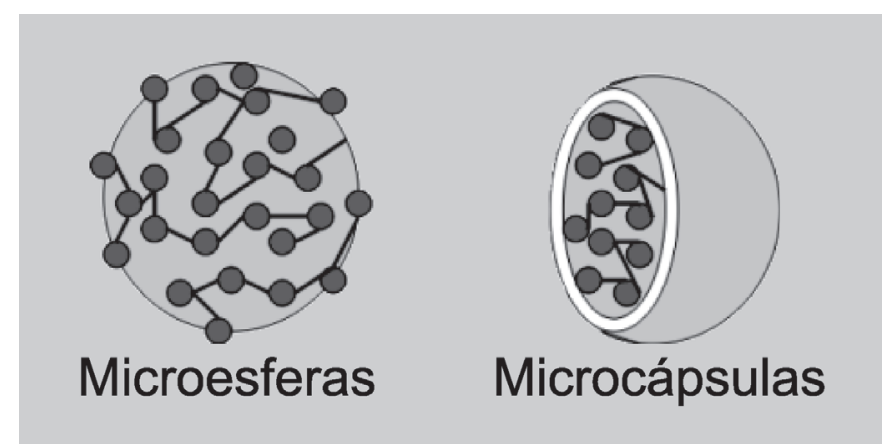

FIGURA 2 - Esquema ilustrativo diferenciando os tipos de micropartículas: microesferas (matriz polimérica) e microcápsulas (cápsulas com parede polimérica e cavidade oca ou aquosa).

vivo, desprovendo o parasita de ferro essencial para seu desenvolvimento (Chabantchick, 1995; Mabeza et al., 1996). O DFO apresenta tempo de meia-vida curto (Keberle, 1964) e a sua biodisponibilidade poderia ser modificada pela microencapsulação. De fato, a formulação de micropartículas apresentou taxa de encapsulamento superior a $70 \%$, perfil cinético caracterizado por rápida liberação inicial, seguida de taxa marginal de liberação controlada.

\section{Vacinas para malária encapsuladas em lipossomas e microesferas}

Pesquisas para o desenvolvimento de vacina eficaz contra a malária estão em andamento. Uma das barreiras a serem vencidas é a associação das vacinas com adjuvantes, que permitam estímulo da resposta imune com níveis de anticorpos elevados por períodos de tempo prolongado (Gupta, 1998; Cui, Mumper, 2003). O uso de técnicas de microencapsulação tem permitido a indução de potente resposta imune humoral, quando modelos de antígenos são utilizados para o desenvolvimento de vacinas (Igartua et al., 1998).

Segundo Moorthy e colaboradores (2004), há aumento no número de grupos de pesquisa na área do desenvolvimento de vacinas eficazes contra a malária, já que novos métodos de liberação controlada da vacina e uso de novos adjuvantes podem elevar os níveis de anticorpos. Os autores argumentam, ainda, que a necessidade de vacina efetiva é urgente e que o seu desenvolvimento é tecnicamente possível na visão da maioria dos pesquisadores.

$\mathrm{O}$ antígeno de merozoíto MAS-2, um candidato à vacina contra a malária, foi testado com o uso de diferentes adjuvantes, FCA/FIA, ISA 720, SAF-1 e LIPO/LA em Alhidroge ${ }^{\circledR}$, utilizando a resposta isolada deste último como padrão. O adjuvante LIPO/LA consiste em lipossomas compostos de dimiristoilfosfatidilcolina, dimiristoilfosfatidilglicerol e colesterol na proporção de 0,9:0,1:0,75. Os resultados mostraram que o uso de LIPO/ LA induziu respostas tão elevadas quanto aquelas baseadas nas formulações de Alhidrogel ${ }^{\circledR}$ isolado (Pye et al., 1997).

Técnicas de nanotecnologia farmacêutica também foram utilizadas para a encapsulação do antígeno recombinante R32NSI em lipossomas, no desenvolvimento de vacina para a malária pelo Instituto de Pesquisa Walter Reed do Exército Americano. Neste projeto, o lipídio monofosforila A foi incluído na bicamada lipídica como adjuvante. $\mathrm{O}$ estudo clínico de fase I em voluntários humanos demonstrou indução promissora de anticorpos antimalária em níveis elevados (Alving, 2002).

A procura de novos adjuvantes para vacinas levou Thomasin e colaboradores (1996) a testar microesferas biodegradáveis de PLGA como adjuvante para um peptídeo sintético da malária com fraco poder imunogênico, o P30B2, em ensaios in vivo utilizando camundongos. Os resultados demonstraram a propriedade das microesferas em induzir a produção de anticorpos e o seu potencial em prolongar os níveis de anticorpos por períodos de até 45 semanas.

Um peptídeo sintético obtido de proteínas do circumesporozoíto de $P$. berghei $(\mathrm{Pb})$ 252-260 foi utilizado em diferentes formulações de microesferas de PLGA, para o estudo da indução de resposta antígeno-específica dos linfócitos T citotóxicos (CTL). Os resultados obtidos de ensaios in vitro e in vivo, utilizando camundongos Balb/ $\mathrm{C}$, indicaram que as microesferas constituem potentes sistemas de liberação de antígenos imunoestimulantes para uma resposta CTL, sendo úteis no desenvolvimento de subunidades de vacinas contra a malária (Men et al., 1997).

Microesferas de PLGA foram utilizadas para encapsular o antígeno sintético SPf66. O objetivo do trabatho foi avaliar a capacidade do SPf66 encapsulado de induzir resposta imune superior àquela de SPf66 administrado com alúmen, um adjuvante tradicional em vacinas. No estudo de imunização in vivo por via subcutânea utilizando camundongos, o antígeno encapsulado induziu maiores níveis de anticorpos anti-SPf66 do que aqueles em que o peptídeo estava adsorvido no alúmen. Segundo os autores, este resultado se deve principalmente à capacidade do sistema em otimizar a penetração do antígeno nas células imunocompetentes (Rosas et al., 2001).

Carcaboso e colaboradores (2003) estudaram o uso de microesferas de PLGA contendo o peptídio sintético SPf66 encapsulado, para a indução de resposta imune protetora parcial contra a malária, utilizando camundongos 
Balb/C. A vacina encapsulada em microesferas foi capaz de induzir, após administração oral, resposta imune sistêmica comparável àquela observada no esquema que utiliza o alúmen por via subcutânea.

Outros polipeptídios candidatos a vacinas contra a malária em desenvolvimento como o RTS/AS02 (Bojang et al., 2001), o Pvs25h (Miles et al., 2002), ou a combinação de MSP1, MSP2, RESA (Genton et al., 2003) estão sendo testados em protocolos tradicionais de imunização.

Edwards e colaboradores (2005) desenvolveram micropartículas de 1-5 $\mu \mathrm{m}$ de diâmetro para administração pulmonar contendo DNA, peptídeos e antígenos da malária, que promoveram a liberação sustentada desses agentes. As partículas degradaram no corpo e liberaram as proteínas MSP-1 e AMA-1 no sangue estimulando a resposta imune humoral. Partículas menores, da ordem de $0,1 \mu \mathrm{m}$ foram preferencialmente fagocitadas pelos APCs, que expressaram as proteínas AMA- 1 and MSP-1 codificadas pelos plasmídeos do DNA, iniciando a resposta imune celular necessária para a imunidade completa.

\section{CONCLUSÕES}

Nos últimos anos, numerosos estudos demonstraram que a distribuição de um fármaco no organismo pode ser modificada pelo uso de nanossistemas tais como lipossomas, nanopartículas ou micropartículas. Estes carreadores podem proteger o princípio ativo da degradação e/ou inativação; melhoram a biodisponibilidade por aumento da penetração celular e proporcionam a liberação do fármaco no sítio de ação desejado, eliminando ou minimizando os efeitos colaterais da terapêutica convencional.

O desenvolvimento de formas farmacêuticas de liberação controlada por micro e nanossistemas poderá permitir um melhor controle da cinética de liberação do fármaco, resultando em níveis plasmáticos terapêuticos, com menores efeitos tóxicos, representando passo importante no desenvolvimento de uma nova terapêutica antimalárica, o que pode repercutir na melhoria da qualidade de vida de milhões de pacientes, além do impulso técnico, cientifico e financeiro, potencialmente adquiridos.

O tratamento da malária pode ser beneficiado através de técnicas de encapsulação de fármacos. Dentre as estratégias de combate à malária utilizando técnicas de nanotecnologia farmacêutica, o desenvolvimento de uma vacina é a alternativa que tem recebido maior atenção dos pesquisadores, com grande número de artigos publicados. Até o presente, escassos estudos foram realizados com o uso de nanodispositivos aplicados ao carreamento de quimioterápicos utilizados no combate à malária. Entretanto, alguns pesquisadores já comprovaram a possibilidade de encapsulação de antimaláricos em lipossomas, nanocápsulas e micropartículas, demonstrando a sua eficiência e utilidade na terapêutica antimalárica.

Os estudos para a obtenção de uma vacina efetiva contra a malária estão avançados e deverá ser liberado nos próximos anos um produto para uso em larga escala. A vacina desenvolvida poderá ser veiculada em nanodispositivos de modo a aumentar a sua estabilidade e prolongar o seu efeito de indução de imunogenicidade. Diversos polipeptídios candidatos a vacinas contra a malária tais como o RTS, S/AS02, o Pvs25H ou a combinação de MSP1, MSP2, e RESA estão sendo testados em protocolos convencionais de imunização e são potenciais candidatos à microencapsulação.

\section{AGRADECIMENTOS}

Os autores agradecem o suporte financeiro parcial do Conselho Nacional de Desenvolvimento Científico e Tecnológico (CNPq), processo 479979/01-4, concedido a NSSM e ao Ministério da Ciência e Tecnologia através da Rede Nacional de Nanobiotecnologia (Nanobiotec)- MCT/ CNPq.

\section{ABSTRACT}

\section{Application of pharmaceutical nanotechnology to the treatment of malaria}

In spite of living in a scientific and technological era, malaria continues to be one of the worldwide greatest health challenges. The state-of-the-art policy to keep malaria under control is expected to comprise jointstrategies, such as the vector control, fast diagnosis, therapeutic guarantee, resistance cutback, drug optimization and development of new therapeutic agents and vaccines. Nano and microcarrier systems have been receiving a special attention, including the development of strategies for carrying bioactive agents, vaccines through nanodevices, such as liposomes and nanoparticles, and microdevices, such as microparticles and microemulsions. Numerous nanosystems have already substantiated their effectiveness to optimize vaccines, insecticides, and chemotherapeutic agents applied to the control of malaria. This review is intended to explain the malaria scenario though the world, and to show the nanotechnology as a promising alternative for malaria control and treatment.

UNITERMS: Malaria. Nanotechnology. Liposomes. Nanoparticles. Microparticles. Vaccines. 


\section{REFERÊNCIAS BIBLIOGRÁFICAS}

AHSAN, F.; RIVAS, I.P.; KHAN, M.A.; TORRES SUAREZ, A.I. Targeting to macrophages: role of physicochemical properties of particulate carriers liposomes and microspheres - on the phagocytosis by macrophages. J. Control. Release, Amsterdam, v.79, p.29-40, 2002.

ALVING, C.R. Design and selection of vaccine adjuvant: animal models and human trials. Vaccine, Amsterdam, v.20, p.S56-S64, 2002.

ANDRADE, C.A.; CORREIA, S.M.T.S.; COELHO, L.C.B.B.; NASCIMENTO, S.C.; SANTOSMAGALHÃES, N.S. Antitumor activity of Cratylia mollis lectin encapsulated into liposomes. Int. J. Pharm., Amsterdam, v.278, p.435-445, 2004.

BHADRA, D.; BHADRA, S.; JAIN, N.K. PEGylated peptide dendrimeric carriers for the delivery of antimalarial drug chloroquine phosphate. Pharm. Res., Arlington, v.23, n.3, p.623-633, 2006.

BARRAT, G.M. Therapeutic applications of colloidal drug carriers. Pharma. Sci. Technol. Today, Cambridge, v.5, p.163-171, 2000 .

BAYOMI, M.A.; AL-ANGARY,A.A.;AL-MESHAL, M.A.; MOHAMED M.; AL-DARDIRI, M.M. In vivo evaluation of arteether liposomes. Int. J. Pharm., Amsterdam, v.175, p.1-7, 1998.

BERGSTRAND, N.; ARFVIDSSON, M.C.; KIM, J.M.; THOMPSON, D.H.; EDWARDS, K. Interactions between $\mathrm{pH}$-sensitive liposomes and model membranes. Biophys. Chem., Amsterdam, v.104, p.361-379, 2003.

BOJANG, K.A.; MILLIGAN, P.J.M.; PINDER, M.; VIGNERON, L.; ALLOUECHE, A.; KESTER, K.E.; BALLOU, W.R.; CONWAY, D.J.; REECE, W.H.H.; GOTHARD, P.; YAMUAH, L.; DELCHAMBRE, M.; VOSS, G.; GREENWOOD, B.M.; HILL, A.; MCADAM, K.P.W.J.; TORNIEPORTH, N.; COHEN, J.D.; DOHERTY, T. Efficacy of RTS,S/AS02 malaria vaccine against Plasmodium falciparum infection in semiimmune adult men in the Gambia: a randomised trial. Lancet, Amsterdam, v.358, p.1927-1934, 2001.
BRIGGER, I.; DUBERNET, C.; COUVREUR, P. Nanoparticles in cancer therapy and diagnosis. Adv. Drug Del. Rev., Arlington, v.54, p.631-651, 2002.

BROSSI, A.; VENUGOPOLAN, B.; DOMENGUER, L.G.; YEH, H.J.C.; FLIPPEN-ANDERSON, J.L.; BUCHS, P.; LUO, X.D.; MILHOUS, W.; PETERS, W. Arteether, a new antimalarial drug: synthesis and antimalarial properties. J. Med. Chem., Washington, v.31, p.645-650, 1988.

CARCABOSO, A.M.; HERNANDEZ, R.M.; IGARTUA, M.; GASCON, A.R.; ROSAS, J.E.; PATARROYO, M.E.; PEDRAZ, J.L. Immune response after oral administration of the encapsulated malaria synthetic peptide SPf66. Int. J. Pharm., Amsterdam, v.260, p.273$282,2003$.

CHABANTCHICK, Z.I. Iron chelators as antimalarials: the biochemical basis of selective cytotoxicity. Parasitol. Today, Amsterdam, v.11, n.2, p.74-78, 1995.

CHEDGZOY, P.; WINCKLE, G.; HEARD, C. M. Triclosan: release from transdermal adhesive formulations and in vitro permeation across human epidermal membranes. Int. J. Pharm., Amsterdam, v.235, p.229-236, 2002.

CHIMANUKA, B.; GABRIËLS, M. DETAEVERNIER, M. R.; PLAIZIER-VERCAMMEN, J. A. Preparation of $\beta$ artemether liposomes, their HPLC-UV evaluation and relevance for clearing recrudescent parasitaemia in Plasmodium chabaudi malaria-infected mice. J. Pharm. Biomed. Anal., Amsterdam, v.28, p.13-22, 2002.

COLTEL, N.; COMBES, V.; HUNT, N.H.; GRAU, G.E. Cerebral malaria - a neurovascular pathology with many riddles still to be solved. Curr. Neurovasc. Res., Amsterdam, v.1, p.91-110, 2004.

CRAWLEY, J. Malaria: new challenges, new treatments. Curr. Paediatrics, Amsterdam, n.9, p.34-41, 1999.

CUI, Z.; MUMPER, R.J. Microparticles and nanoparticles as delivery systems for DNA vaccines. Crit. Rev. Ther. Drug Carrier Syst., Redding, v.20, n.2-3, p.103-137, 2003.

DIAS, L.R.S. Situação mundial da malária. Infarma, v.14, p.87-88, 2002. 
DIEBOLD, Y.; JARRÍN, M.; SÁEZ, V.; CARVALHO, E.L.S.; OREA, M.; CALONGE, M.; SEIJO, B.; ALONSO, M.J. Ocular drug delivery by liposomechitosan nanoparticle complexes (LCS-NP). Biomaterials, Amsterdam, v.28, n.8, p.1553-1564, 2006.

DUNNE, M.; BIBBY, D.C.; JONES, J.C.; CUDMORE, S. Encapsulation of protamine sulphate compacted DNA in polylactide and polylactide-co-glycolide microparticles. $J$. Control. Release, Amsterdam, v.92, p.209-219, 2003.

EDWARDS, D.A.; SUNG, J.; PULLIAM, B.; WEHRENBERG-KLEE, E.; SCHWARTZ, E.; DREYFUSS, P.; KULKARNI, S.; LIEBERMAN, E. Pulmonary delivery of malarial vaccine in the form of particulates. PCT Int. Appl. WO 2005110379, 2005. 25 p.

FLORENS, L.; WASHBURN, M.P.; RAINE, J.D.; ANTJONY, R.M.; GRAINGER, M.; HAYNES, J.D.; MOCH, J.K.; MUSTER, N.; SACCI, J.B.; TABB, D.L.; WITNEYA.A.; WOLTERS, D.; WU, Y.; GARDNER, M.; HOLDER, A.A.; SINDEN R.E.; YATES, J.R.; CARUCCI, D.J. A proteomic view of the Plasmodium falciparum life cicle. Nature, London, v.419, p.520-526, 2002.

FOLEY, M.; TILLEY, L. Quinoline antimalarials: Mechanisms of action and resistance and prospects for new agents. Pharmacol. Ther., Amsterdam, v.79, p.55-87, 1998.

FUNDAÇÃO NACIONAL DE SAÚDE(FUNASA), 2003. Disponível em: <www.funasa.gov.br>. Acesso em: 12 jan. 2004.

FUNDAÇÃONACIONAL DE SAÚDE(FUNASA). Manual de terapêutica da malária. Brasília, 2001. 104 p.

FUNDAÇÃO NACIONAL DE SAÚDE (FUNASA). Programa Nacional de Prevenção e Controle da Malária - PNCM. Brasília, 2002. 44p.

FUNDAÇÃO NACIONALDE SAÚDE(FUNASA). Ações de Controle de Endemias - Malária. Brasília, 2002b. $104 \mathrm{p}$.

GABRIËLS, M.; PLAIZIER-VERCAMMEN, J. Physical and chemical evalutation of liposomes, containing artesunate. J. Pharm. Biomed. Anal., Arlington, v.31, p.655-667, 2003.
GENTON, B.; AL-YAMAN, F.; BETUELA, I.; ANDERS, R. F.; SAUL, A.; BAEA, K.; MELLOMBO, M.; TARAIKA, J.; BROWN, G.V.; PYE, D.; IRVING, D.O.; FELGER, I.; BECK, H.P.; SMITH, T.A.;ALPERS, M.P. Safety and immunogenicity of a three-component bloodstage malaria vaccine (MSP1, MSP2, RESA) against Plasmodium falciparum in Papua New Guinean children. Vaccine, Amsterdam, v.22, p.30-41, 2003.

GOMES, A.P.; SIQUEIRA-BATISTA, R.; GONÇALVES, M. L. C.; IGREJA, R. P. In: Rodrigo Siqueira Batista, Andréia patrícia Gomes, Ricardo Pereira Igreja \& Donald William Huggins, Medicina tropical. Abordagem atual das doenças infecciosas e parasitárias. São Paulo: Cultura Médica, 2001. v.1, cap.17, p.150-166.

GREF, R.; MINAMITAKE, Y.; PERACCHIA, M.T.; TRUBETSKOY, V.; TORCHILIN, V.; LANGER, R. Biodegradable long-circulating polymeric nanospheres. Science, New York, v.263, p.1600-1603, 1994.

GUPTA, R.K. Aluminum compounds as vaccine adjuvants. Adv. Drug Del. Rev., Arlington, v.32, p.155-172, 1998.

IGARTUA, M.; HERNANDEZ, R.M.; ESQUISABEL, A.; GASCON, A.R.; CALVO, M.B.; PEDRAZ, J.L. Enhanced immune response after subcutaneous and oral immunization with biodegradable PLGA microspheres. $J$. Control. Release, Amsterdam, v.56, p.63-73, 1998.

KEBERLE, $\mathrm{H}$. The biochemistry of desferrioxamine and its relation to iron metabolism. Annal. New York Acad. Sci., New York, v.119, p.758 - 768, 1964.

KREMSNER, P.G; KRISHNA, S. Antimalarial combinations. Lancet, Amsterdam, v.364, p.285-294, 2004.

KRETTLI, A.U.; ANDRADE-NETO, V.F.; BRANDAO, M.G.; FERRARI, W.M.S. The search for new antimalarial drugs from plants used to treat fever and malaria or plants randomly selected: a review. Mem. Inst. Oswaldo Cruz, Rio de Janeiro, v.96, p.1033-1042, 2001.

KUMAR, V.; ABBAS, A.K; FAUSTO, N. Patologia - Bases patológicas das doenças. 7 ed. Rio de Janeiro: Ed. Saunders Elsevier, 2005. p.357-432.

LASIC, D.D. Novel application of liposomes. Trends Biotechnol., Amsterdam, v.16, p.307-321, 1998. 
LEITE, E.A.; GRABE-GUIMARÃES, A.; GUIMARÃES, H.N.; MACHADO-COELHO G.L.L.; BARRATT G.; MOSQUEIRA, V.C.F. Cardiotoxicity reduction induced by halofantrine entrapped in nanocapsule devices. Life Sci., Amsterdam, v.80, p.1327-1334, 2007.

LEGRAND, P.; BARRATT, G.; MOSQUEIRA, V.; FESSI, H.; DEVISSAGUET, J-P. Polymeric nanocapsules as drug delivery systems: a review. S.T.P. Pharma Sci., Paris v.9, p.411-418, 1999.

LOISEAU, P.M.; LE BRAS, J. New drugs against parasitic diseases. Rev. Prat., Paris, v.57, p.175-182, 2007.

LONGMUIR, K.J.; ROBERTSON, R.T.; HAYNES S.M.; BARATTA, J.L.; WARING A.J. Effective targeting of liposomes to liver and hepatocytes in vivo by incorporation of a Plasmodium amino acid sequence. Pharm. Res., Arlington, v.23, p.759-769, 2006.

MABEZA, G.F.; BIEMBA, G.; GORDEUK, V.R. Clinical studies of iron chelators in malaria. Acta Haematol., Amsterdam, v.95, p.78-86, 1996.

MANDAL, T.K.; BOSTANIAN, L.A.; GRAVES, R.A.; CHAPMAN, S.R.; IDODO, T.U. Porous biodegradable microparticles for delivery of pentamidine. Eur. J. Pharm. Biopharm., Amsterdam, v.52, p.91-96, 2001.

MEN, Y.; TAMBERT, H.; AUDRAN, R.; GANDER, B.; CORRADIN, G. Induction of a cytotoxic T lymphocyte response by immunization with a malaria specific CTL peptide entrapped in biodegradable polymer microspheres. Vaccine, Amsterdam, v.15, n.12/13, p.1405-1412, 1997.

MILES,A.P.;ZHANG Y.; SAUL,A.; STOWERS, A.W. Largescale purification and characterization of malaria vaccine candidate antigen pvs $25 \mathrm{~h}$ for use in clinical trials. Protein Express. Purification, Amsterdam, v.25, p.87-96, 2002.

MOGHIMI, S.M.; HUNTER, A.C.; MURRAY, J.C. Nanomedicine: current status and future prospects. The FASEB J., v.19, p.311-330, 2005.

MOORTHY, V.S.; GOOD, M.F.; HILL, A.V.S. Malaria vaccine developments. Lancet, Amsterdam, v.363, p.150156, 2004.
MOSQUEIRA, V.C.F.; LOISEAU, P.M.; BORIES, C.; LEGRAND, P.; DEVISSAGUET, J.; BARRATT, G. Efficacy and pharmacokinetics of intravenous nanocapsule formulations of halofantrine in Plasmodium berghei-infected mice. Antimicrob. Agents Chemother., Washington, v.48, p.1222-1228, 2004.

MOSQUEIRA, V.C.F.; LEGRAND, P.; BARRATT, G. Surface-modified and conventional nanocapsules as novel formulation for parenteral delivery of halofantrine. $J$. Nanosci. Nanotechnol., Stevenson Ranch, v.9-10, p.3193-3202, 2006.

PONTES, A.C.O.; CAETANO, M.N.P.; SANTOSMAGALHÃES, N.S. Physicochemical characterization and antimicrobial activity of benzathine penicillin $\mathrm{G}$ liposomes. S.T.P. Pharma Sci., Paris, v.9, p.419-427, 1999.

PYE, D.; VANDENBERG, K.L.; DYER, S.L.; IRVING, D.O.; GOSS, N.H.; WOODROW, G.C.; SAULI, A.; ALVING, C.R.; RICHARDS, R.L.; BALLOU, W.R.; WU, M.; SKOFF, K.; ANDERSO, R.F. Selection of an adjuvant for vaccination with the malaria antigen, MSA2. Vaccine, Amsterdam, v.15, n.9, p.1017-1023, 1997.

RIBEIRO-COSTA, R. M.; PEREIRA, E.C.G.; ALVES, J.A.; SANTOS, N.P.; NASCIMENTO, S. C.; HONDA, N.K.; SILVA, N.H.; SANTOS-MAGALHÃES, N.S. In vitro and in vivo properties of usnic acid encapsulated into PLGA-microspheres. J. Microencap., London, v.21, p.371-384, 2004.

ROSAS, J.E.; HERNÁNDEZ, R.M.; GASCÓN, A.R.; IGARTUA, M.; GUZMAN, F.; PATARROYO, M.E.; PEDRAZ, J.L. Biodegradable PLGA microspheres as a delivery system for malaria synthetic peptide SPf66. Vaccine, Amsterdam, v.19, p.4445-4451, 2001.

SAKATA, S.; UCHIDA, K.; KAETSU, I.; KITA, Y. Programming control of intelligent drug releases in response to single and binary environmental stimulation signals using sensor and electroresponsive hydrogel. Radiat. Phys. Chem., Amsterdam, v.76, p.733-737, 2007.

SANTOS, N.P.; NASCIMENTO, S.C.A.; SILVA, J.F.; PEREIRA, E.C.G.; SILVA, N.H.; HONDA, N.K.; SANTOS-MAGALHÃES, N.S. Usnic acid-loaded nanocapsules: an evaluation of cytotoxicity. J. Drug Del. Sci. Tech., Paris, v.15, p.355-361, 2005. 
SAPRA, P.; ALLEN, T. M. Ligant-target liposomal anticancer drugs. Prog. Lipid Res., Amsterdam, v.42, p.439-462, 2003.

SCHLICHER, E.J.A.M.; POSTMA, N.S.; ZUIDEMA, J.; TALSMA, H.; HENNINK, W.E. Preparation and characterisation of Poly(D,L-lactic-co-glycolic acid) microspheres containing desferrioxamine. Int. J. Pharm., Amsterdam, v.153, p.235-245, 1997.

SILVA, G.A. Introduction to nanotechnology and its applications to medicine. Surgical Neurol., Amsterdam, v.61, p.216-220, 2004.

TAO, S.L.; DESAI, T.A. Microfabricated drug delivery systems: from particles to pores. Adv. Drug Del. Rev., Arlington, v.55, p.315-328, 2003.

THOMASIN, C.; CORRADIN, G.; MEN, Y.; MERKLE, H.P.; GANDER, B. Tetanus toxoid and synthetic malaria antigen containing poly(lactide)/poly(lactide-co glycolide) microspheres: importance of polymer degradation and antigen release for immune response. $J$. Control. Release, Amsterdam, v.41, p.131-145, 1996.

TRACY, J.W.; WEBSTER JÚNIOR, L.T. Fármacos usados na quimioterapia das infecções por protozoários: malária. In: Goodman \& Gilman. As bases farmacológicas da terapêutica. 10 ed. Rio de Janeiro: Mc Graw Hill, 2003. cap.40 p.803-822.

TRACY, J.W.; WEBSTER JÚNIOR, L.T. Fármacos usados no tratamento das protozoonoses: malária. In: Goodman \& Gilman. As bases farmacológicas da terapêutica. 9 ed, Rio de Janeiro: McGraw-Hill, 1996. cap.40, p.709-724.
VANDENBERG, G.W.; DROLET, C.; SCOTT S.L.; DE LA NOUE, J. Factors affecting protein release from alginatechitosan coacervate microcapsules during production and gastric/intestinal simulation. J. Control. Release, Amsterdam, v.77, p.297-307, 2001.

VERMA, R.K.; GARG, S. Current status of drug delivery technologies and future directions. Pharmac. Technol., v.25, n.2, p.1-4, 2001.

WILAIRATANA, P.; KRUDSOOD, S.; TREEPRASERTSUK, S.; CHALERMRUT, K.; LOOAREESUWAN, S. The future outlook of antimalarial drugs and recent work on the treatment of malaria. Arch. Med. Res., Amsterdam, v.33, p. 416-421, 2002.

WINSTANLEY, P.O. Modern chemotherapeutic options for malaria. Lancet, Amsterdam, v.1 p. 242-250, 2001.

WONGSRICHANALAI， C.; PICKARD， A.L.; WERNSDORFER, W.H.; MESHNICK, S.R.O. Epidemiology of drug-resistant malaria. Lancet Inf. Dis., Amsterdam, v.2, p.209-218, 2002.

YOUAN, B.B.C.; JACKSON, T.L.; DICKENS, L.; HERMANDEZ, C.; OWUSUBIOABA, G. Protein release profiles and morphology of biodegradable microcapsules containing an oil core. J. Control. Release, Amsterdam, v.76, p.313-326, 2001.

Recebido para publicação em 07 de agosto de 2006. Aceito para publicação em 08 de agosto de 2007. 